\title{
The European Southern Observatory
}

\author{
A. Blaauw \\ Director General of ESO
}

The European Southern Observatory, ESO, is an organization for astronomical research. It is a joint enterprise of European astronomers aimed at conducting research at a level beyond that which can be reached by the scientific institutes and manpower of the individual Member States. These States are Belgium, Denmark, France, the Federal Republic of Germany, the Netherlands and Sweden.

In the southern part of the Atacama desert in Chile ESO already operates its observatory with modern astronomical instrumentation of medium size. This distant site was chosen for several reasons: first of all in view of the outstanding weather conditions, but also because the ESO astronomers are particularly interested in the study of a number of celestial objects visible from the southern hemisphere which have no counterpart in the northern sky.

A battery of telescopes is in use. One of them serves mainly for astronomical spectroscopy, it is equiped with a light gathering mirror of $1.5 \mathrm{~m}$ diameter and various spectrographs. Another telescope serves for photometric measures, i.e. for observing the intensity of the star light by photoelectric methods in the integrated wavelength areas the astronomer wishes to study; it has a mirror of $1 \mathrm{~m}$ diameter and is equiped with various kinds of photometers. Also in operation is a double astrograph, i.e. twin telescopes using objective lenses of $40 \mathrm{~cm}$ diameter, one of which is equiped with an objective prism of special design and constructed for the wholesale measurement of stellar velocities through the Doppler shift. A large Schmidt telescope, equal in size to that of the Palomar observatory in California, is now under construction and expected to be in operation by the middle of 1971 .

\section{Large Telescope}

ESO's main instrument, however, will be a telescope of exceedingly large light gathering power with a mirror of $3.6 \mathrm{~m}$ diameter. It was meant to be the largest telescope in the southern hemisphere, far surpassing in size the largest one now in operation (in South Africa). After the ESO project was initiated by European astronomers, plans for large southern telescopes of about equal size were also proposed, and are being realized, by groups in the USA and in the United Kingdom.

A telescope of this size presents problems of design and construction of a new order of magnitude. It, moreover, must be equiped with auxiliary instrumentation of a complexity comparable to that of the telescope proper. Hence, this telescope requires a considerable amount of new developments in the fields of optics, mechanical design, electronics, and automation. There are, moreover, interesting interface problems between the telescope and the building that houses it.

The engineering experience, technical know-how, and administrative apparatus built up by CERN for handling projects of this size, led the Council of ESO to seek CERN's collaboration at this moment, at which ESO wishes to pursue with the utmost strength the realization of its principal instrument. Details of an agreement for this purpose have been worked out by the Directorates and Councils of the two Organizations and were formally approved by both Councils in June of this year. Under the terms of the agreement an "ESO Telescope Division" will be set up at CERN. It will have financial independence of CERN with responsibility for the development of the properties of the telescope exclusively to the ESO Directorate, but, in respect to administrative set-up and staff regulations, it will be largely adapted to CERN.

\section{The Objects of Research}

Objects for study of particular interest are the Magellanic Clouds and the central regions of the Galaxy (the Milky Way stellar system). The Magel-

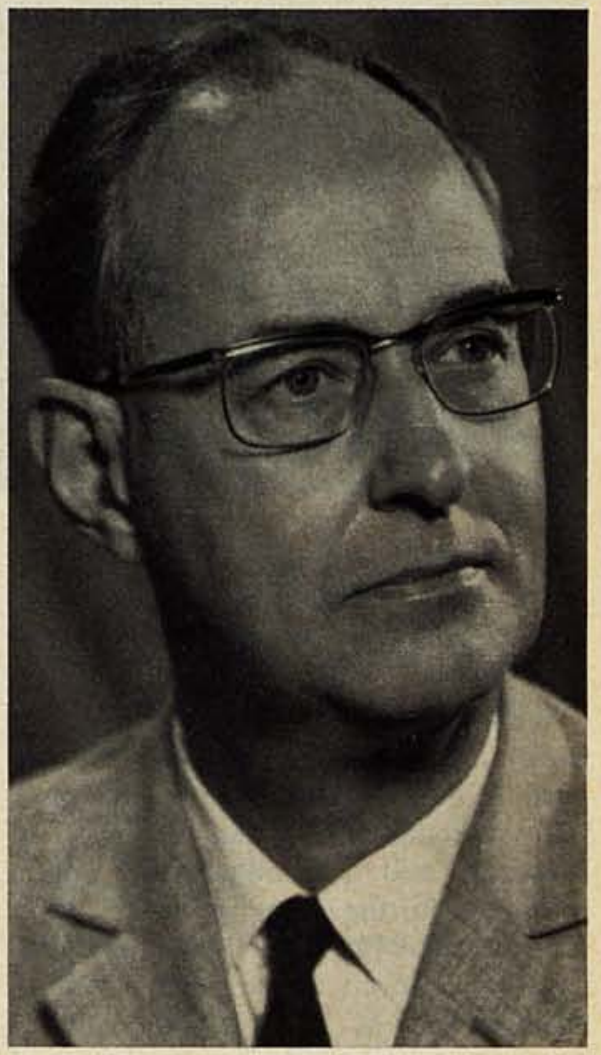

lanic clouds are stellar systems outside the Galaxy - probably satellites of it - and can be seen only from the southern hemisphere. They are ten times nearer to us than the Andromeda nebula, the stellar system which for northern hemisphere astronomers is the principal object for extragalactic studies. Thus, the Magellanic Clouds are in a much more favourable position. They contain both very old and quite young stars and are still the scene of active star formation. They are therefore, for studies of the process of star birth and of stellar evolution, a magnificent natural laboratory.

The central region of the Galaxy also a typical southern object - is of great importance for the study of galactic structure. We know, from scarce observations so far in our Galaxy, but from more extensive information derived from other stellar systems, that in the central parts of the large spiral nebulae violent physical processes occur which are of 


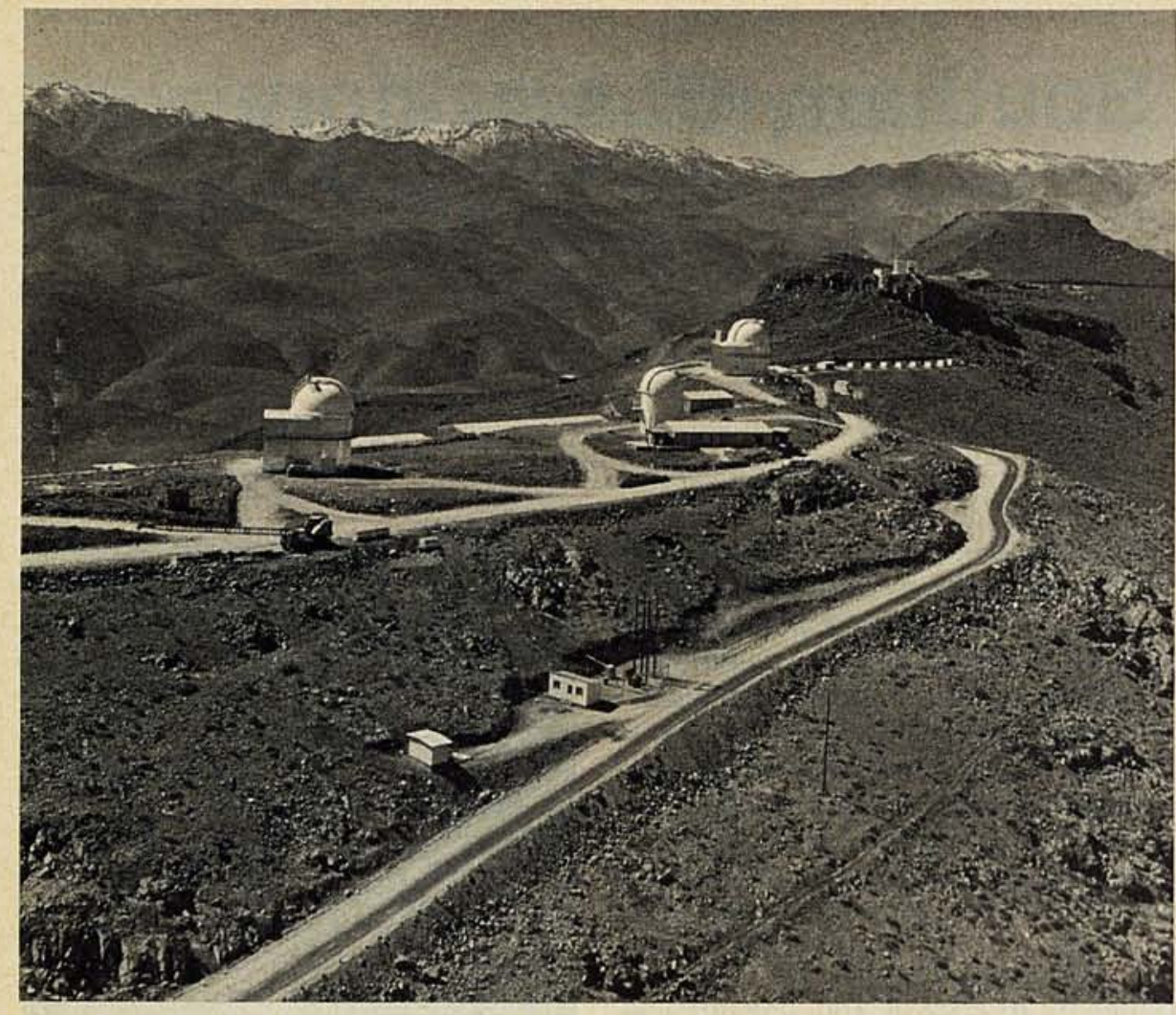

fundamental importance for the outer parts of the system and which must give valuable clues to their evolutionary history.

Whereas these two fields of research were the principal inducement for putting the observatory on the southern hemisphere, a new direction of research will probably draw much attention in the coming years; it is connected with high energy astrophysics. Recent discoveries in both radio-astronomy and optical astronomy have pointed to the existence of phenomena dominated by high energy physical processes. We observe these in the form of quasars and sources of high frequency radiation. We are entering here a field of great common interest to astronomers and high energy physicists, which may open fascinating prospects for scientific collaboration between ESO and CERN.

\section{The Nature and Structure of ESO}

ESO is governed by a Council, now under the presidency of Mr. J.H. Bannier of The Netherlands, in which each of the Member States is represented by two delegates, usually one astronomer and one government official. The Organization's affairs are conducted by the Director General. He delegates, for the operation of the observatory in Chile, responsibility for the daily affairs to the Director in Chile, Prof. B.E. Westerlund, and is in technical matters assisted by Prof. J. Ramberg. The ESO Telescope Division at CERN will be, at least pro- visionally, under the daily supervision of Dr. S. Laustsen.

Several advisory committees assisted the ESO Council and its Directorate - the Instrumentation Committee under chairmanship of Prof. $\mathrm{Ch}$. Fehrenbach of France; the Finance Committee, chairman Dr. C. Zelle of the Federal Republic of Germany; and the Scientific Programs Committee, chairman Prof. B. Strömgren of Denmark.

Although already more than a decade has elapsed since the initiative to create ESO was taken, activities in Chile started only fairly recently. In the early years extensive site tests were conducted in the South African desert region, but their results turned out not to be quite as promising as those discovered in the meantime in Chile. On the basis of a multilateral agreement between ESO and that country, constructions began in Chile in 1964, under the former Director General, Prof. O. Heckman and the superintendant in Chile, Dr. A.B. Muller.

ESO built its Chilean Headquarters in Santiago, the capital of Chile, and the observatory proper on the mountain La Silla. These Headquarters contain the administrative offices and the permanent facilities for the scientific staff: offices, main library, measuring apparatus, computing facilities. Also, the main workshop and laboratories (electronics, optical) are to be found there. Most of the scientific staff live with their families in Santiago and divide their time for research between the observatory on
Aerial photograph of the ESO Observatory on the mountain La Silla in the Atacama desert in Chile. Near the right hand is the already flattended summit on which is to be erected the $3.6 \mathrm{~m}$ telescope. In the foreground are the domes housing the already operating telescopes. Off the picture to the left is the hostel, where the stalf is lodged.

(Photos ESO)

La Silla and the Santiago Headquarters.

The observatory is located about $600 \mathrm{~km}$ north of Santiago, i.e. a day's travel by plane, car or bus. Through the collaboration of the Chilean Government, ESO acquired a property of about $630 \mathrm{~km}^{2}$ of desert area, large enough to safeguard it from building or mining activities which might disturb the astronomical observations by the production of dust or light.

After the completion of the construction of almost $40 \mathrm{~km}$ of road, leading to the summit of La Silla at an altitude of $2400 \mathrm{~m}$, the present telescope buildings as well as a hostel for the lodging of astronomers, technical staff and administrative personnel were erected, including the many auxiliary facilities like power supply, water supply, heating plant etc., which are needed for such an isolated establishment in the desert. This first phase of construction in Chile was completed early in 1969, and on March 25, 1969, its official dedication by the President of the Republic, Eduardo Frei Montalva, took place on La Silla.

The European Central Office of ESO is at Hamburg. Here are the principal offices of the Director General, the Technical Director and the Head of Administration. This Office maintains the relations with, on the one hand the astronomers and the governments of the Member States, and on the other hand with the observatory in Chile and from now on with Telescope Division at CERN.

The accompanying aerial view of $\mathrm{La}$ Silla shows, in the foreground, the array of telescope buildings, workshops etc. and in the background the summit of La Silla, already flattened for the erection of the $3.6 \mathrm{~m}$ telescope. For its successful realization, hopefully within the next five years, ESO now looks forward to fruitful collaboration of all those whose contributions will be involved: the ESO Telescope Division at CERN, the CERN expertise and experience, the ESO astronomical staff in Chile, and the ESO Instrumentation Committee. 